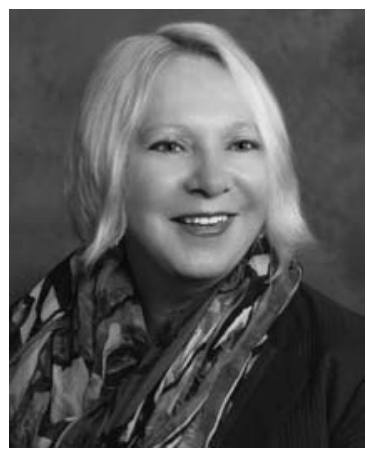

\title{
A Conversation with Valerie Weaver
}

\author{
Interviewer: ElizaBeth McKenNA \\ Associate Editor, Cancer Discovery
}

Valerie Weaver is a Professor in the Departments of Surgery, Bioengineering and Therapeutic Sciences, Anatomy, and Radiation Oncology and the Director of the Center for Bioengineering and Tissue Regeneration at the University of California, San Francisco.

Elizabeth McKenna: Can you tell us a little bit about the role of forces in cancer progression?

Dr. Weaver: The way that I view cancer is that, in terms of the mechanics, it's a physical alteration of the tissue on multiple levels. On a gross level, you have a large cell mass surrounded by a dense extracellular matrix that is also highly vascularized. Tumors contain a lot of fluid in part because the vasculature is altered and lymphatic drainage is often compromised. The fibrotic tumor mass and high interstitial pressure can create a milieu where it's really hard to get drugs in. That is certainly an aspect of tumor mechanics that has been studied a lot and what most people think about when they discuss mechanics. What my group has been looking at, and what people haven't really thought about as much, is that tumor cells also alter their behavior in response to the tissues mechanics and the genomic alterations in the tumor cells changes their contractility behavior. Many common oncogenes change both how squishy the cells are and how much the cells can pull on each other and on the matrix around them. A highly contractile tumor cell will remodel the matrix around itself. The contractility of the tumor cell will also alter how the cell responds to exogenous signals like growth factors. It also determines the kind of soluble factors that the tumor cells will secrete and we believe that this is, in part, some of what ultimately will stimulate some of the stromal cells-including infiltrating immune cells - as well as the fibroblasts. The fibroblasts themselves are super-synthetic. They create and secrete a lot of matrix proteins that they lay down and remodel, and these matrix proteins create a denser environment that is a bit stiffer. They also have enzymes that cross-link that matrix and remodel it, and they can even generate aligned matrix bundles that act like migration tracks along which tumor cells, immune cells, and even fibroblasts can migrate. A lot of cytokines are also being constantly secreted within a tumor that can also stimulate the fibroblasts to synthesize and remodel the matrix and these cytokines will attract immune cells to come into the tumor-and they also get in on the action by secreting things like TGF- $\beta$, which additionally drives the fibroblasts to produce even more matrix proteins that ultimately create this really dense, stiff, fibrotic tissue. The induction of the stiff, fibrotic tissue has repercussions across the board in terms of the physical properties of the environment and how the tumor cells, the immune cells, and the vasculature are influenced by that stiffness to change their behavior and responsiveness to soluble factors.

What people also don't think about is that, as the tumor mass expands, it's not expanding like a balloon into infinite space. It's expanding into this really dense fibrotic environment. Of course, the dense environment resists. You have a solid stress from the tumor cells as they expand. You have a reciprocal tension from this dense environment. You have a buildup of fluid, so you ultimately have this massive solid stress in the tumor. All of these conspire to create an environment that is really difficult to get drugs into. It creates a hypoxic environment. This also changes the tumor's behavior as well by fundamentally reprograming how that tumor cell behaves.

The key point is that a tumor is not mechanically homogeneous. It's not like one big ball of a stiff tumor. It's a very heterogeneous physical environment. You have little tiny soft niches interspersed with thick matrix bundles that act like little railroad tracks where the cells migrate on these stiff tracks. It's a really complicated and fascinating thing to try to understand how these forces add yet another layer of complexity to the tumor, and how they change the initiation, progression, and aggressiveness of the cancer.

Elizabeth McKenna: You've spoken about intrinsic and extrinsic forces. Can you elaborate about that and the differences between the two types of forces?

Dr. Weaver: Every cell in your body, even if it's not a muscle cell, has myosin. Cells are contractile. That's how I define intrinsic tension. "Force" has a time element and

(C) 2016 Weaver. This article is distributed under the terms of the Creative Commons Attribution-NonCommercial License, which permits reuse and redistribution, except for commercial purposes, provided that the original author and source are credited. 
is often represented as stress which is measured as force over area. It's important to realize that it is an active process. Cells are continuously interrogating their microenvironment. They'll either pull on each other or they'll pull on the matrix. That's intrinsic tension. In a tumor or in a normal cell, if you put them in a stiff environment they tune their tension to the best of their capacity to the stiffness of the environment around them or to the stiffness of the other cells that they're interacting with. In a tumor, we know that there are genetic modifications. What's really interesting is that many of the same genetic pathways that are selected for in cancer are same ones that alter intrinsic tension in the cell. Of course, if you have a big mass of cells and it's pushing out, that's also a type of intrinsic force.

One type of extrinsic mechanical property in a tumor is the stiffness of the extracellular microenvironment. Another type of extrinsic or external force is the shear force that a tumor cell would experience when it enters into the vasculature. Other types of extrinsic forces include interstitial pressure, as well as any long-range stresses such as tissue distortion or stretch. What is important to realize is that extrinsic forces in a tissue cross length scales from a subcellular level to a tissue level. For instance, there is a gross "organ and tissue-level" length scale that affects how the cell and the tissue behaves that can influence the direction of fluid flow or perhaps the alignment of collagen fibers or even the vasculature within the tumor. However, what the cell really cares about is what's happening right around it. That determines how it signals and how it behaves given the various different soluble factors that it's simultaneously experiencing.

Elizabeth McKenna: How do the tumor cells sense the force? What molecules are involved in sensing? How do they propagate the signal to effect changes in the phenotype?

Dr. Weaver: It's not as though you have specialized mechanotransducers, although in some instances you do; some ion channels, for example, have been known for decades as being sensitive to mechanical cues. Cilia are quintessential mechanosensors. Hearing is basically mediated through ion channels because cilia are sensitive to change induced by sound waves and they open up and regulate the ion channels. That's one way to sense mechanical cues. Integrins also are classic mechanotransducers. An integrin responds to the mechanics of the environment, but it also responds to different extracellular matrix proteins, so it's also a biochemical signal. Biochemistry and biomechanics are different sides of the same coin.

Virtually any transmembrane receptor that can change its conformation in response to a strain could be viewed as a mechanoreceptor - in the case of integrins, talin and vinculin are sensitive to force, which favors their open configuration to favor binding to the integrin tail and to each other. For a protein to be considered a mechanosensor, the molecule must be able to respond to force by altering its structure/function, whether this is opening up a cryptic site, enhancing the timing of the opening of an ion channel to allow in ions, or closing an ion channel: any of these things will function as a mechanosensor. Then something has to be able to amplify that response, but even then it doesn't do anything unless it can actually effect a change, either by changing actin polymerization, by changing higher-order chromatin structure so it modifies the next signal, or by just amplifying the signal. All of those things will eventually affect how the cell responds.

Elizabeth McKenna: Do all tumor cells react to forces and interact with their extracellular matrix in the same way, or does it depend on their genetic background?

Dr. Weaver: It depends. It's something that we're just starting to scratch the surface of. A lot of people in this engineering and biophysics field are looking for certain principles that we can be guided by to think about how tissue mechanics can be layered on top of everything that we know about how tumors develop and behave. Having said that, if you think about a glioblastoma versus a pancreatic cell versus a bone, each of these cells has fundamentally different mechanobehaviors to begin with. The kind of mechanical cues and how they're going to change the cell's behavior are probably going to be slightly different. The kind of pathways that would be affected would be slightly different because they're different to begin with, but there could be similar principles that we're working across.

Certainly, our data with the pancreas suggest that if you have a more basal genotype in the tumor you have a much more contractile phenotype and enhanced mechanobehavior: a very unique mechanical signature. We also have some new data on glioblastomas that, if you have an isocitrate dehydrogenase mutation that is known to create a much lower grade or less aggressive tumor, it curbs or curtails the mechanophenotype. Those are two independent things that say that it's different. Triple-negative breast cancers versus luminal breast cancers have fundamentally different mechanics. Maybe it's a continuum with different molecules that affect things differently, but ultimately higher or lower stiffness affecting how the tumor behaves.

Elizabeth McKenna: Do you have any ideas on ways that we could design drugs that could target certain pathways that are involved in mechanotransduction, or should we be thinking about targeting the stroma and affecting the components of the extracellular matrix?

Dr. Weaver: A naïve perspective would be to say, "Oh, we're just going to go in and inhibit mechanics." The problem is, ROCK ( $\rho$-associated protein kinase) inhibitors are contraindicated for treatment of brain tumors because they cause a massive drop in blood pressure. If you just completely ablate the stroma, certain of the tumor cells are really contractile to begin with, so they won't care. That could explain why there have been problems. Plus, the stroma has good things that it does, not just bad things.

We're looking at three or four different approaches. One: Can we exploit aspects of the mechanics and the things that we know about mechanics to enhance patient 
stratification? If we can identify those patients, for example, that have a very unique mechanical signature, maybe they would be contraindicated for some of the stromal ablation therapies. Or those individuals might have tumors with a higher propensity to metastasize, so those individuals would be treated differently. That's how we're thinking about it for pancreatic cancer.

We're also doing some studies with other people where we're looking at lipophilic statins, which have effects on protein prenylation. They calm down the tension in the tumor. Those individuals that are on these lipophilic statins have a lower incidence, or at least less aggressive, pancreatic cancer. They have a longer expected survival. Those are ways to think about it.

We've also developed mass spectrometry methods to look at the profile of crosslinks in the matrix. Those would then tell us which enzymes are changing the crosslinking. If those are unique in tumors, we could target those. Lysyl oxidase is one, but it's not good for pancreas because the propeptide has anti-Ras effects. It could work for other kinds of tumors, but not for a Ras-dependent tumor. There are also prolyl hydroxylases out there, so that's another way to think about it. I know Sunil Hingorani is leading an effort to reduce solid stress by treating pancreatic patients with hyaluronidase - this is a fantastic approach-although how this truly works remains unclear. Finally, understanding how a mechanical change in a tumor drives its dysfunction could identify unique molecular targets that could be the tumor's Achilles' heel.

Another factor to consider is whether an altered tumor mechanics alters immune function. We have active studies exploring this interesting area-could we enhance the antitumor immune response by normalizing tumor mechanics? Clearly, only one approach will be insufficient, and it will be important to explore this issue using a lot of different angles. We will have to work as a team with our colleagues who are doing fantastic work in immune manipulation. We're also doing some really cool studies with biophysicists to understand how ECM mechanics regulate at the nanolevel, how Ras signaling occurs, and how RTK (receptor tyrosine kinase) signaling is processed in the cell. This collaborative work is yielding some really interesting insights and key targets that we could use. It turns out that the whole dynamics of Ras signaling and effector recruitment and turnover are exquisitely sensitive to the mechanical cues in the environment. The devil is in the details. We need to go after this in a lot of different ways. A really simplistic perspective is not going to work. I would say that's what got us into these problems in the first place. I'd rather use a scalpel than a hatchet.

Elizabeth McKenna: There'll probably be a lot of context specificity and maybe different combination of strategies will be needed.

Dr. Weaver: When I first started doing this, colleagues of mine in cancer biology thought I was a flake. Now I'm pleased to say that - maybe not in the mainstream cancer field, but certainly in the engineering and biophysicsthey're all going crazy mad on this stuff. They think that it's going to be one-size-fits-all, and it's all going to be supersimple. I'm coming along, and of course they don't want to hear this, but I think, "No, no, no. It's not like that." It's actually more elegant and more interesting. 


\section{$\$_{\text {CSH\& }}^{\infty}$ Cold Spring Harbor Symposia SYMPOSIA}

\section{A Conversation with Valerie Weaver}

Cold Spring Harb Symp Quant Biol 2016 81: 354-356 originally published online April 19, 2017 Access the most recent version at doi:10.1101/sqb.2016.81.032128

Creative This article is distributed under the terms of the

Commons http://creativecommons.org/licenses/by-nc/4.0/, which permits reuse and

License redistribution, except for commercial purposes, provided that the original author and source are credited.

Email Alerting Receive free email alerts when new articles cite this article - sign up in Service the box at the top right corner of the article or click here. 